\title{
Article \\ Performance Analysis of a Multi-Function Mach-Zehnder Interferometer Based Photonic Architecture on SOI Acting as a Frequency Shifter
}

\author{
Gazi Mahamud Hasan*(D), Mehedi Hasan and Trevor J. Hall $\mathbb{D}$ \\ Photonic Technology Laboratory, Centre for Research in Photonics, University of Ottawa, 25 Templeton St., \\ Ottawa, ON K1N 7N9, Canada; mhasa067@uottawa.ca (M.H.); Trevor.Hall@uottawa.ca (T.J.H.) \\ * Correspondence: ghasa102@uottawa.ca
}

Citation: Hasan, G.M.; Hasan, M.; Hall, T.J. Performance Analysis of a Multi-Function Mach-Zehnder Interferometer Based Photonic Architecture on SOI Acting as a Frequency Shifter. Photonics 2021, 8 , 561. https://doi.org/10.3390/ photonics 8120561

Received: 9 November 2021 Accepted: 6 December 2021 Published: 9 December 2021

Publisher's Note: MDPI stays neutral with regard to jurisdictional claims in published maps and institutional affiliations.

Copyright: (c) 2021 by the authors. Licensee MDPI, Basel, Switzerland. This article is an open access article distributed under the terms and conditions of the Creative Commons Attribution (CC BY) license (https:// creativecommons.org/licenses/by/ $4.0 /)$.

\begin{abstract}
A photonic frequency shifter based on generalized Mach-Zehnder interferometer (GMZI) architecture is presented and experimentally validated. The circuit consists of four Mach-Zehnder modulators (MZM) in a $4 \times 4$ network bounded by two $4 \times 4$ multimode interference couplers and functionally equivalent to two parallel dual-parallel MZM (DP-MZM). The circuit can offer static bias free operation, virtual connectivity control of the components, and spatial separation of upand down-converted carriers, which can be collected from separate ports without using any optical demultiplexing filters. Thus, the design permits remote heterodyning (advantages which cannot be obtained using a commercial DP-MZM or filter based optical frequency shifter). Experimental investigation shows deviation from ideal performance due to possible fabrication error and poor fiber-chip coupling. A carrier suppression of $>20 \mathrm{~dB}$ and spurious sideband suppression $>12 \mathrm{~dB}$ relative to the principal harmonics is achieved without any tuning for bias adjustment. In addition to the frequency conversion, the integration feasible circuit can also perform as a sub-carrier generator, IQ modulator, and frequency multiplier.
\end{abstract}

Keywords: generalized Mach-Zehnder interferometer; photonic frequency shifter; silicon on insulator (SOI); multimode interference coupler; Si-based DP-MZM; multi-functional photonic integrated circuit

\section{Introduction}

Wireless communication has undergone rapid evolution due to exponential growth in data traffic and demand of super broadband services. Congested lower frequency bands and costly, complicated, and power-hungry high frequency operations dictate the wireless access network to adopt other means in meeting the challenge of ubiquitous connection among the end-users with energy efficiency. Hall et al. argued that in cluttered urban environments, a digital coherent radio-over-fiber $(\mathrm{RoF})$ link backed by distributed antenna system is a viable energy efficient solution to address the evolution of broadband wireless access systems [1]. For its effective realization, efficient single sideband (SSB) modulation utilizing a photonic frequency shifter at the uplink is vital [2]. Photonic frequency conversion has also become an integral part of applications such as frequency-shifted feedback (FSF) lasers [3], high-resolution laser spectroscopy [4], multicarrier generation [5,6], true time delay beam steering in frequency domain continuous wave (FMCW) rada rs [7], Doppler lidar systems [8], and ultra-high-Q photonic crystal nanocavities [9].

Diverse methods such as serrodyne translation [8,10], TE/TM mode conversion [11], stimulated Brillouin scattering [12,13], and acousto-optic deflection [14,15] have been adopted to realize a high-performance frequency shifter. The serrodyne technique can offer high spectral purity, but demands that the modulator and driving circuit have a very high bandwidth. Low frequency limited acousto-optic approaches requires delicate phase matching. All-optical frequency shifting offered by stimulated Brillouin scattering is limited by the fixed Brillouin frequency of a fiber and a complicated pump wave locking 
procedure. For heterodyne light communication systems, the conventional choice is an OSSB modulator followed by filter structure to isolate the desired shifted band [16-19]. The frequency response of the filter and temperature control to maintain alignment among the source, modulator, and filter make the conversion procedure complicated and power hungry. To the best of the authors' knowledge, an IQ modulator based filterless integrated frequency shifter was first introduced by Izutsu et al. [20]. After that, numerous investigations on Mach-Zehnder interferometric IQ modulator-based frequency conversion techniques have been reported in the scientific literature [21-29]. Most of these circuits employ single $\mathrm{LiNbO}_{3}$ dual-parallel Mach-Zehnder modulator (DP-MZM) based architecture realizing single sideband-suppressed carrier (SSB-SC) modulation [20-24]. The conventional $\mathrm{LiNbO}_{3}$ modulator can offer high extinction ratio, high bandwidth, and linear electro-optic effect, but it suffers from high drive voltage and severe dc bias drift due to charge screening and dielectric relaxation. In addition, the $\mathrm{LiNbO}_{3}$ platform is not suitable for large-scale photonic integration. The CMOS compatible Si platform can offer a compact footprint. Recent demonstrations of single DP-MZM based frequency converters on Si platform show impressive performance in terms of suppression of unwanted sidebands [26,27]. All of these circuits are optimized for one output which results in either up-conversion or down-conversion to be achieved at a time. Yamazaki et al. utilized a $2 \times 2$ coupler instead of a Y-junction at the output side of the DP-MZM to spatially separate the first order up- and down-converted frequency components [23,24]. Hasan et al. proposed a two-stage series parallel MZM architecture which is capable of spatial separation of higher order frequency components [28]. Spatially separated frequency shifting operation offers simultaneous up- and down-conversion which translates to coherent sub-carrier generation and separation without any need of an inflexible optical demultiplexing filter. Two DP-MZMs configured in parallel have also been applied to frequency shifting [30-32]. While these architectures offer high order frequency conversion, the employment of a polarization division multiplexing scheme render their integration feasibility complicated.

In this report, an experimental demonstration of a generalized Mach-Zehnder interferometerbased architecture capable of frequency conversion is presented. The circuit consists of four parallel differentially driven MZMs connected in an interferometer structure bounded by two $4 \times 4$ multimode interference (MMI) couplers. The circuit is functionally equivalent to two parallel DP-MZMs with a lower coupler count: two $4 \times 4$ couplers are needed in the outer stages instead of six $2 \times 2$ couplers [32]. The intrinsic phase relationship of MMI couplers enables a static bias free operation; all MZMs are biased at null point and each functionally equivalent DP-MZM is biased at its quadrature point by design, and thus the DC bias drift problem can be avoided. Spatial separation between the up- and down-converted signals is obtained simultaneously which can also be applied as coherent subcarrier generation scheme without multiplexing filter. The hardwired connections from the MZMs to the outer-stage MMI couplers and the default null point biasing offer a mechanism of withdrawing any MZM from the operation without physically disconnecting it (a degree of freedom much desired for a photonic integrated circuit). The circuit is fabricated on silicon-on-insulator (SOI) platform in a multi-project wafer (MPW) facility using 'off the shelf' components. Wideband operation of MMI coupler supported by ideal fabrication process can lead to a tuning-free, temperature insensitive operation. An experiment is conducted to validate the theoretical prediction of its ability to shift the carrier frequency by the modulating frequency. A series of tests have also been conducted to identify the fabrication errors and their effects on the performance of the circuit. Although the discussion presented in this report is focused on its frequency shifting capability, the circuit can offer other functionalities such as complex modulation, frequency multiplication, sub-carrier generation, and more.

The remainder of this paper is organized as follows. In Section 2, the theoretical framework of operating principle of the circuit under discussion is provided and validation by simulation is presented. Section 3 provides information on device fabrication and 
structure. Section 4 presents the experimental setup and analyzes the measured results. Finally, the work is summarized in Section 5.

\section{Theory}

The conventional waveguide-based frequency shifter follows the principle of single sideband modulation (SSB). A dual parallel Mach-Zehnder modulator (DPMZM) with null-point biased differentially driven MZMs on its two arms and the outer Mach-Zehnder interferometer (MZI) structure biased at its quadrature point can perform the SSB frequency electro-optical up-conversion function [20]. The structure is basically an I-Q modulator with an RF electrical signal is applied to the I channel and a $\pi / 2$ phase-shifted replica of the same RF electrical signal is applied to the $Q$ channel. Figure 1 shows the circuit and its corresponding output spectrum showing the carrier shifted by the first harmonic of the RF drive frequency. Spurious side-harmonics can be suppressed effectively by restricting operation in small signal modulation range at the expense of lower conversion efficiency [26]. Figure $1 \mathrm{~b}$ shows the carrier's shift to upper sideband (USB) which can be switched to lower sideband (LSB) by simply changing the polarity of the phase in the outer MZI.
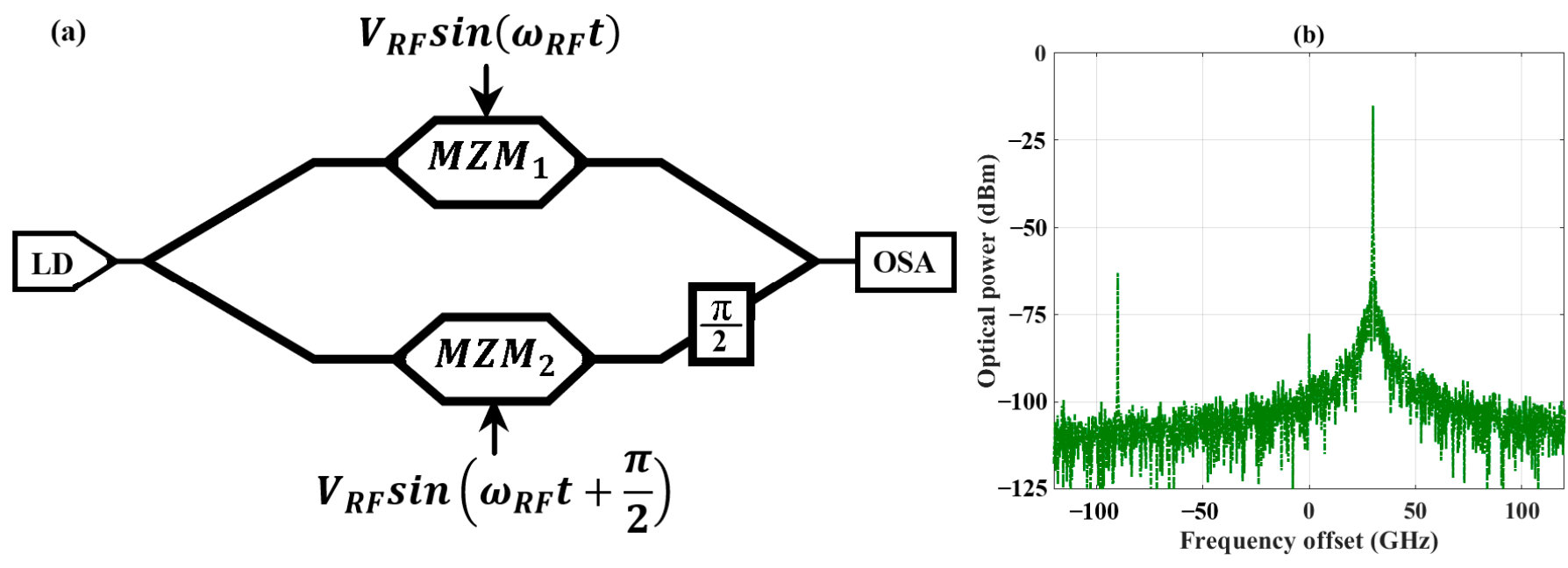

Figure 1. (a) Schematic diagram of conventional optical frequency shifter; (b) optical spectrum of the circuit showing the up-converted carrier shifted by RF frequency $30 \mathrm{GHz}$. Simulation is carried out using Virtual photonics Inc. (VPI) software package. MZM, Mach-Zehnder modulator; OSA, optical spectrum analyzer; LD, laser diode.

In principle, the photonic circuit reported here consists of two IQ modulators in parallel. The configuration supports simultaneous down- and up-conversion while spatially separating them. Figure 2 depicts the proposed circuit which consists of four differentially driven MZMs in the intermediate stage of a $4 \times 4$ network. The outer stages providing the function of distribution and combination of signals are realized by two $4 \times 4 \mathrm{MMI}$ couplers, respectively. Two $2 \times 2 \mathrm{MMI}$ couplers are used for connecting each pair of phase modulators of the individual MZI structure, as shown in Figure 2. 


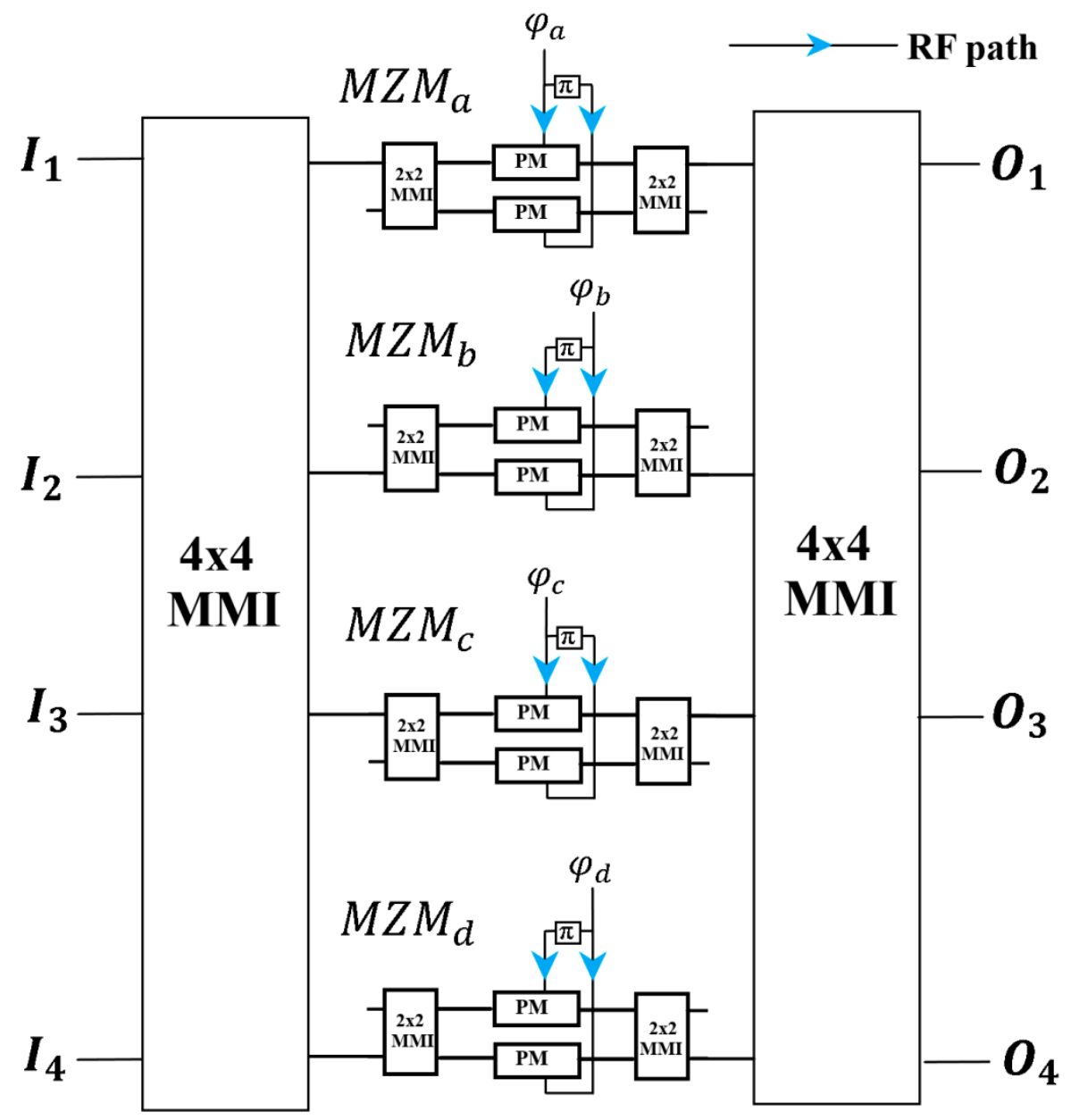

Figure 2. Schematic diagram of the photonics frequency shifter circuit. Each MZM is driven differentially with modulating signals $\varphi_{a}, \varphi_{b}, \varphi_{c}$ and $\varphi_{d}$, respectively. PM, phase modulator; MMI, multimode interference coupler.

Figure 2 shows that each MZM has one of the ports at its input-output couplers unused. The intrinsic relative phase relationships between the ports of MMI coupler and the choice of input-output ports enable each individual MZM to be biased at its minimum transmission point (MITP) by design. The transfer matrix of an individual MZM can be expressed as:

$$
\left[\begin{array}{l}
b_{1} \\
b_{2}
\end{array}\right]=T_{2 \times 2}\left[\begin{array}{cc}
\exp \left(i \varphi_{1}\right) & 0 \\
0 & \exp \left(i \varphi_{2}\right)
\end{array}\right] T_{2 \times 2}\left[\begin{array}{l}
a_{1} \\
a_{2}
\end{array}\right]
$$

where $T_{2 \times 2}=\frac{1}{\sqrt{2}}\left[\begin{array}{cc}1 & -i \\ -i & 1\end{array}\right]$ is the transfer matrix of a $2 \times 2$ MMI coupler and $\varphi_{n}$ is the phase shift applied to the $n$th arm of an MZM. Based on the input/output ports of each MZM chosen and the phase shift applied to drive each MZM differentially, the transmission function of each MZM is transformed to:

$$
f_{\mathrm{MZM}_{n}}=i \sin \left(\varphi_{n}\right)
$$

To realize the framework of two parallel DP-MZMs, each functionally equivalent to the circuit shown in Figure 1a, the quadrature bias for individual DP-MZM is provided by 
the intrinsic relative phase relationships among the ports of the $4 \times 4 \mathrm{MMI}$ couplers at the outer stages. The total transfer matrix of the architecture can be expressed as:

$$
\left[\begin{array}{c}
O_{1} \\
O_{2} \\
O_{3} \\
O_{4}
\end{array}\right]=i T_{4 \times 4}\left[\begin{array}{cccc}
\sin \left(\varphi_{a}\right) & 0 & 0 & 0 \\
0 & \sin \left(\varphi_{b}\right) & 0 & 0 \\
0 & 0 & \sin \left(\varphi_{c}\right) & 0 \\
0 & 0 & 0 & \sin \left(\varphi_{d}\right)
\end{array}\right] T_{4 \times 4}\left[\begin{array}{c}
I_{1} \\
I_{2} \\
I_{3} \\
I_{4}
\end{array}\right] .
$$

where

$$
T_{4 \times 4}=\frac{1}{\sqrt{4}}\left[\begin{array}{cccc}
1 & -\zeta & \zeta & 1 \\
-\zeta & 1 & 1 & \zeta \\
\zeta & 1 & 1 & -\zeta \\
1 & \zeta & -\zeta & 1
\end{array}\right] \quad ; \quad \zeta=e^{i \pi / 4}
$$

is the transfer matrix of a $4 \times 4 \mathrm{MMI}$ coupler.

It is possible to achieve different configurations where each DP-MZM has different sets of MZMs as its constituents by varying the modulating signal. Figure 2 shows one example which retains a mirror symmetry in terms of circuit connection. If two modulating signals $V_{1}=V_{I 1}+i V_{Q 1}$ and $V_{2}=V_{I 2}+i V_{Q 2}$ are applied in such a way that $\varphi_{a}=\pi V_{I 1} / v_{\pi}$ $\varphi_{b}=\pi V_{Q 1} / v_{\pi}, \varphi_{c}=\pi V_{Q 2} / v_{\pi}$ and $\varphi_{d}=\pi V_{I 2} / v_{\pi}$, the upper two MZMs form one IQ modulator and the lower two MZMs form the other one. Assuming only $I_{1}$ to be connected to the optical source, the outputs of the architecture can be expressed as:

$$
\left[\begin{array}{l}
O_{1} \\
O_{2} \\
O_{3} \\
O_{4}
\end{array}\right]=\frac{i}{4}\left[\begin{array}{c}
\sin \left(\pi V_{I 1} / v_{\pi}\right)+i \sin \left(\pi V_{Q 1} / v_{\pi}\right)+\sin \left(\pi V_{I 2} / v_{\pi}\right)+i \sin \left(\pi V_{Q_{2}} / v_{\pi}\right) \\
\zeta\left\{-\sin \left(\pi V_{I 1} / v_{\pi}\right)-\sin \left(\pi V_{Q_{1}} / v_{\pi}\right)+\sin \left(\pi V_{I 2} / v_{\pi}\right)+\sin \left(\pi V_{Q_{2}} / v_{\pi}\right)\right\} \\
\zeta\left\{\sin \left(\pi V_{I 1} / v_{\pi}\right)-\sin \left(\pi V_{Q_{1}} / v_{\pi}\right)-\sin \left(\pi V_{I 2} / v_{\pi}\right)+\sin \left(\pi V_{Q_{2}} / v_{\pi}\right)\right\} \\
\sin \left(\pi V_{I 1} / v_{\pi}\right)-i \sin \left(\pi V_{V_{1}} / v_{\pi}\right)+\sin \left(\pi V_{I 2} / v_{\pi}\right)-\sin \left(\pi V_{Q_{2}} / v_{\pi}\right)
\end{array}\right]\left[I_{1}\right]
$$

For $\left(V_{I 1} / v_{\pi}, V_{Q 1} / v_{\pi}, V_{I 2} / v_{\pi}, V_{Q 2} / v_{\pi}\right) \ll 1$, Equation (4) can be written as:

$$
\left[\begin{array}{c}
O_{1} \\
O_{2} \\
O_{3} \\
O_{4}
\end{array}\right] \approx \frac{i \pi}{4 v_{\pi}}\left[\begin{array}{c}
V_{1}+V_{2} \\
\zeta\left\{\left(V_{I 2}-V_{I 1}\right)+\left(V_{Q 2}-V_{Q 1}\right)\right\} \\
\zeta\left\{\left(V_{I 1}-V_{I 2}\right)+\left(V_{Q 2}-V_{Q 1}\right)\right\} \\
V_{1}{ }^{*}+V_{2}{ }^{*}
\end{array}\right]\left[I_{1}\right]
$$

where $\left(^{*}\right)$ stands for complex conjugate. In the case of a pure tone modulating signal, i.e., $\varphi_{a}=\varphi_{d}=m \cos \left(\omega_{R F} t\right)$ and its companion $\pi / 2$ phase shifted replica, i.e., $\varphi_{b}=\varphi_{c}=$ $m \cos \left(\omega_{R F} t+\pi / 2\right)$, the outputs of the architecture can be expressed as:

$$
\begin{gathered}
{\left[\begin{array}{l}
O_{1} \\
O_{2} \\
O_{3} \\
O_{4}
\end{array}\right]=\left[\begin{array}{c}
-i \sum_{n=1}^{\infty} J_{2 n-1}(m)\left\{(-1)^{n} \cos \left[(2 n-1) \omega_{R F} t\right]+i \sin \left[(2 n-1) \omega_{R F} t\right]\right\} \\
0 \\
0 \\
-i \sum_{n=1}^{\infty} J_{2 n-1}(m)\left\{(-1)^{n} \cos \left[(2 n-1) \omega_{R F} t\right]-i \sin \left[(2 n-1) \omega_{R F} t\right]\right\} \\
\Rightarrow
\end{array}\right]\left[I_{1}\right]} \\
\\
{\left[\begin{array}{l}
O_{1} \\
O_{2} \\
O_{3} \\
O_{4}
\end{array}\right]=\left[\begin{array}{c}
i\left\{J_{1}(m) \exp \left[-j \omega_{R F} t\right]-J_{3}(m) \exp \left[j 3 \omega_{R F} t\right] \ldots\right\} \\
0 \\
0 \\
i\left\{J_{1}(m) \exp \left[j \omega_{R F} t\right]-J_{3}(m) \exp \left[-j 3 \omega_{R F} t\right] \ldots\right\}
\end{array}\right]\left[I_{1}\right]}
\end{gathered}
$$

It can be observed from Equation (6) that an electrical to optical frequency up-conversion is achieved with the lower optical sideband available from output port $O_{1}$ and the upper optical sideband available from output port $\mathrm{O}_{4}$. Figure 3 shows the shifted optical spectrum for a RF drive frequency of $10 \mathrm{GHz}$. It can be observed that all even order harmonics, including the carrier are suppressed. Among the odd order harmonics, for output port $O_{1}$, positive orders equal to $(2 p+1)$, with $p$ even, and negative orders equal to $-(2 p+1)$, with $p$ odd, are suppressed and vice versa for output port $O_{4}$. Interchanging the local 
oscillator setting, i.e., $\varphi_{a}=\varphi_{d}=m \cos \left(\omega_{R F} t+\pi / 2\right)$ and $\varphi_{c}=\varphi_{b}=m \cos \left(\omega_{R F} t\right)$ leads the LSB-USB operation to swap their respective output ports, maintaining their distinct spatial separation. Flexibility in choosing other output ports for LSB-USB operations can also be obtained by simply choosing other input ports for optical carrier.

(a)

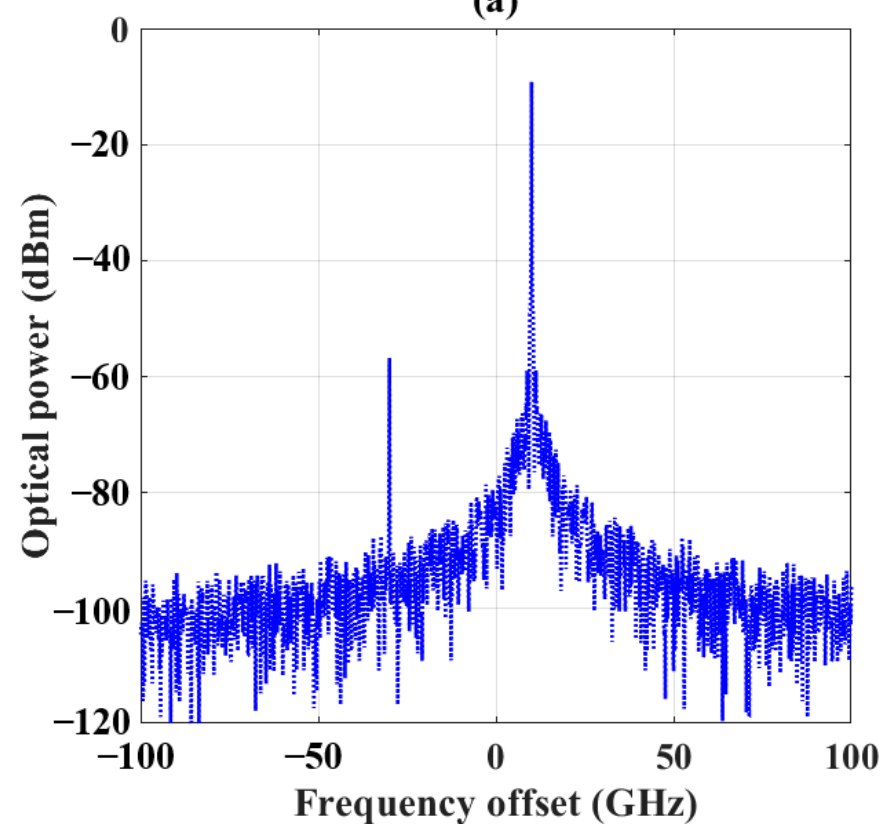

(b)

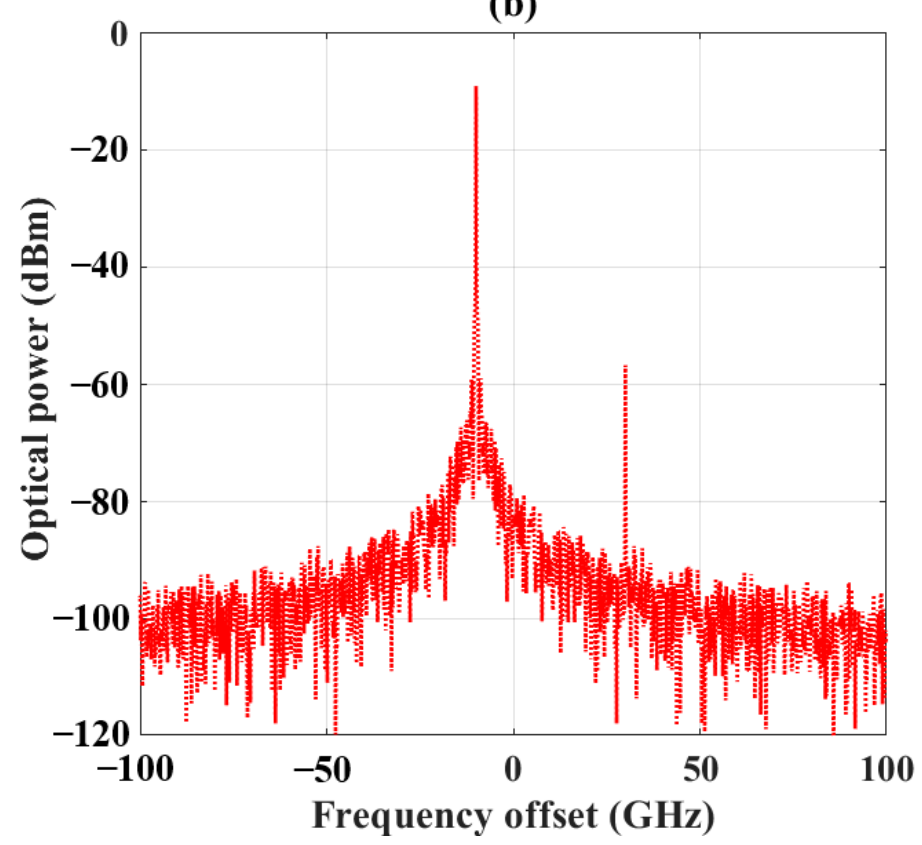

Figure 3. Optical spectra due to (a) frequency up-conversion, and (b) frequency down-conversion. The RF frequency and reference frequency are $10 \mathrm{GHz}$ and $193.4125 \mathrm{THz}$, respectively. Simulation is carried out using Virtual photonics Inc. (VPI) software package.

The circuit offers a mechanism for removing any MZM from the operation without physically disconnecting it; identical phase shift at both arms forces the MZI to act as a cross-over. Due to the unused cross-ports as shown in Figure 2, any MZI can be cut-off completely at the expense of optical power. For example, assigning $\varphi_{c}=\varphi_{d}=0$ leads the removal of the lower IQ modulator. Thus, Equation (3) is modified to:

$$
\left.\begin{array}{c}
{\left[\begin{array}{l}
O_{1} \\
O_{2} \\
O_{3} \\
O_{4}
\end{array}\right]=i T_{4 \times 4}\left[\begin{array}{cccc}
\sin \left(\varphi_{A}\right) & 0 & 0 & 0 \\
0 & \sin \left(\varphi_{B}\right) & 0 & 0 \\
0 & 0 & \sin \left(\varphi_{C}\right) & 0 \\
0 & 0 & 0 & \sin \left(\varphi_{D}\right)
\end{array}\right] T_{4 \times 4}\left[I_{1}\right]} \\
{\left[\begin{array}{l}
O_{1} \\
O_{2} \\
O_{3} \\
O_{4}
\end{array}\right]=\frac{1}{2}\left[\begin{array}{c}
-i \sum_{n=1}^{\infty} J_{2 n-1}(m)\left\{(-1)^{n} \cos \left[(2 n-1) \omega_{R F} t\right]+i \sin \left[(2 n-1) \omega_{R F} t\right]\right\} \\
e^{i 3 \pi / 4} \sum_{n=1}^{\infty} J_{2 n-1}(m)\left\{(-1)^{n} \cos \left[(2 n-1) \omega_{R F} t\right]+\sin \left[(2 n-1) \omega_{R F} t\right]\right\} \\
-e^{i 3 \pi / 4} \sum_{n=1}^{\infty} J_{2 n-1}(m)\left\{(-1)^{n} \cos \left[(2 n-1) \omega_{R F} t\right]-\sin \left[(2 n-1) \omega_{R F} t\right]\right\} \\
-i \sum_{n=1}^{\infty} J_{2 n-1}(m)\left\{(-1)^{n} \cos \left[(2 n-1) \omega_{R F} t\right]-i \sin \left[(2 n-1) \omega_{R F} t\right]\right\}
\end{array}\right]\left[I_{1}\right]} \\
O_{1} \\
O_{2} \\
O_{3} \\
O_{4}
\end{array}\right]=\frac{1}{2}\left[\begin{array}{c}
i\left\{J_{1}(m) \exp \left[-j \omega_{R F} t\right]-J_{3}(m) \exp \left[j 3 \omega_{R F} t\right] \ldots\right\} \\
\sqrt{2} e^{i 3 \pi / 4}\left\{-J_{1}(m) \cos \left(\omega_{R F} t+\pi / 4\right)+J_{3}(m) \cos \left(3 \omega_{R F} t-\pi / 4\right)\right\} \\
\sqrt{2} e^{i 3 \pi / 4}\left\{J_{1}(m) \cos \left(\omega_{R F} t-\pi / 4\right)-J_{3}(m) \cos \left(3 \omega_{R F} t+\pi / 4\right)\right\} \\
i\left\{J_{1}(m) \exp \left[j \omega_{R F} t\right]-J_{3}(m) \exp \left[-j 3 \omega_{R F} t\right] \ldots\right\}
\end{array}\right]\left[I_{1}\right]
$$

Both Equations (6) and (7) offer a spatial separation of lower and upper optical firstorder harmonics. Proper selection of RF amplitude can suppress third order harmonics. The convenience of spatially separated coherent carrier facilitates remote heterodyning 
for wireless access network using a digital coherent RoF system. One of the two firstorder harmonics can be modulated by a second IQ modulator and the two harmonics combined and propagated through the optical fiber. After its transmission to a high-speed photodetector at the remote antenna, the two harmonics beat to produce a modulated RF carrier with a center frequency equal to the double of the RF frequency.

\section{Device Fabrication}

A top-view microscope image of the frequency up-converter is shown in Figure 4. Device fabrication was performed using the $A^{*}$ Star Institute of Microelectronics (IME) CMOS compatible process on a SOI wafer. The SOI wafer has a top Si thickness of $220 \mathrm{~nm}$ and buried oxide thickness of $2 \mu \mathrm{m}$ [33]. It can be observed that path-length matching is a critical design objective due to the larger length of the electro-optic modulator stack in comparison to the I/O-end-MMIs. A racetrack method has been applied where concentric circles are used to ensure that each lane encounters compensating short and long paths around bends. Each phase modulator is realized by a reverse biased p-n diode. An efficient mechanism for coupling of light to and from the circuit at the edges of the chip is absent.

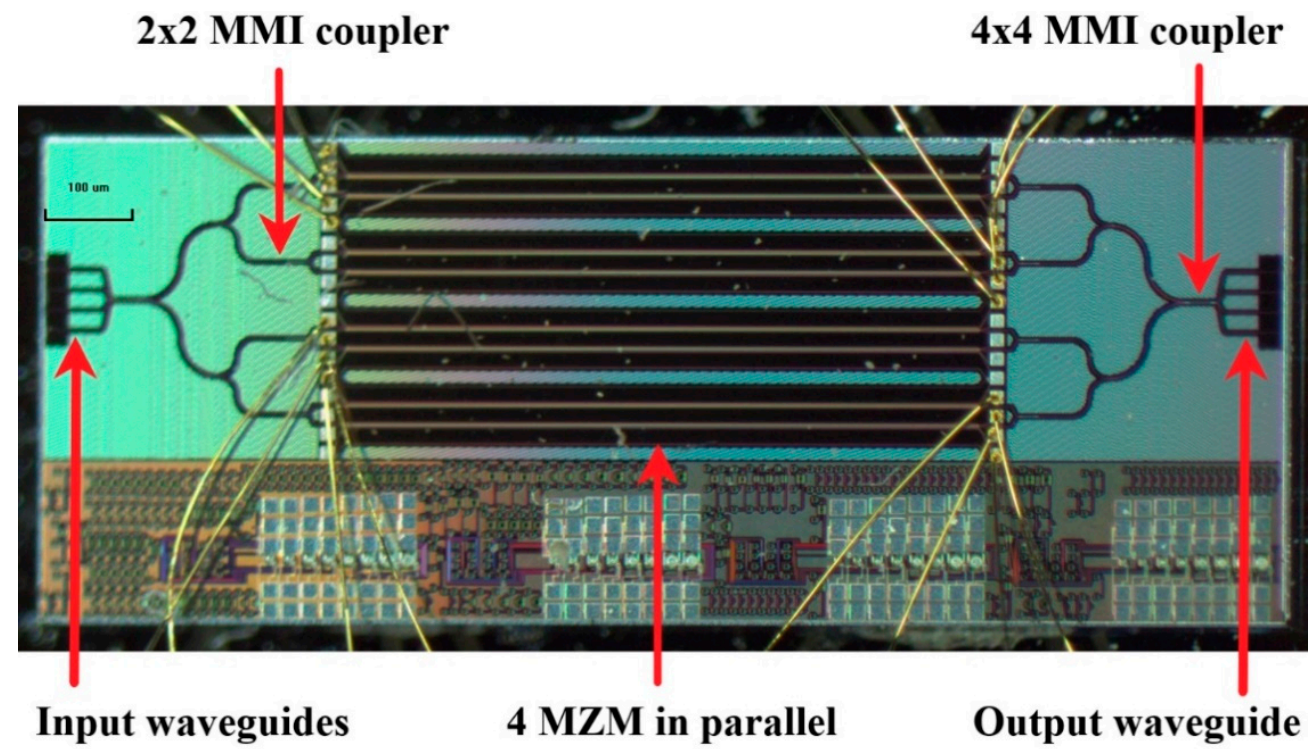

Figure 4. Top-view microscopic image of the fabricated circuit.

\section{Experimental Result and Discussion}

Figure 5 depicts the experimental setup of the fabricated frequency shifter. The built-in laser diode of an optical modulation analyzer (Agilent N4391A, Keysight Technologies, Santa Rosa, CA, USA). provides the optical input to the circuit. The same OMA is used to analyze the output and thus, coherency is maintained. A polarization controller (PC) followed by a polarization beam-splitter (PBS) is used to maintain TE polarization input to the chip. The other port of the PBS is attached to an optical power meter (Anritsu ML910B, Anritsu Corporation, Atsugi, Japan) which is used to monitor the power of the TM component. By adjusting the PC, the TM component can be minimized and the stability of the polarization state of the input light can be observed and maintained. Lensed fibers are used for input-output coupling. A digital storage oscilloscope (Infiniium DSO-2091604A, Keysight Technologies, Santa Rosa, CA, USA) is used after the OMA. For dynamic operation, four RF drive signals with phase sequence shown in Figure 5 are taken from an arbitrary waveform generator (Fluke 294, Fluke Corporation, Everett, WA, USA). A combination of $1 \times 2 \mathrm{RF}$ splitter and a $180^{\circ} \mathrm{RF}$ phase shift is used to differential drive each pair of phase modulators. A DC signal is also applied via a bias tee to provide the voltage required for the reversed-biased $p$-n junction operation of individual optical phase shifters. 


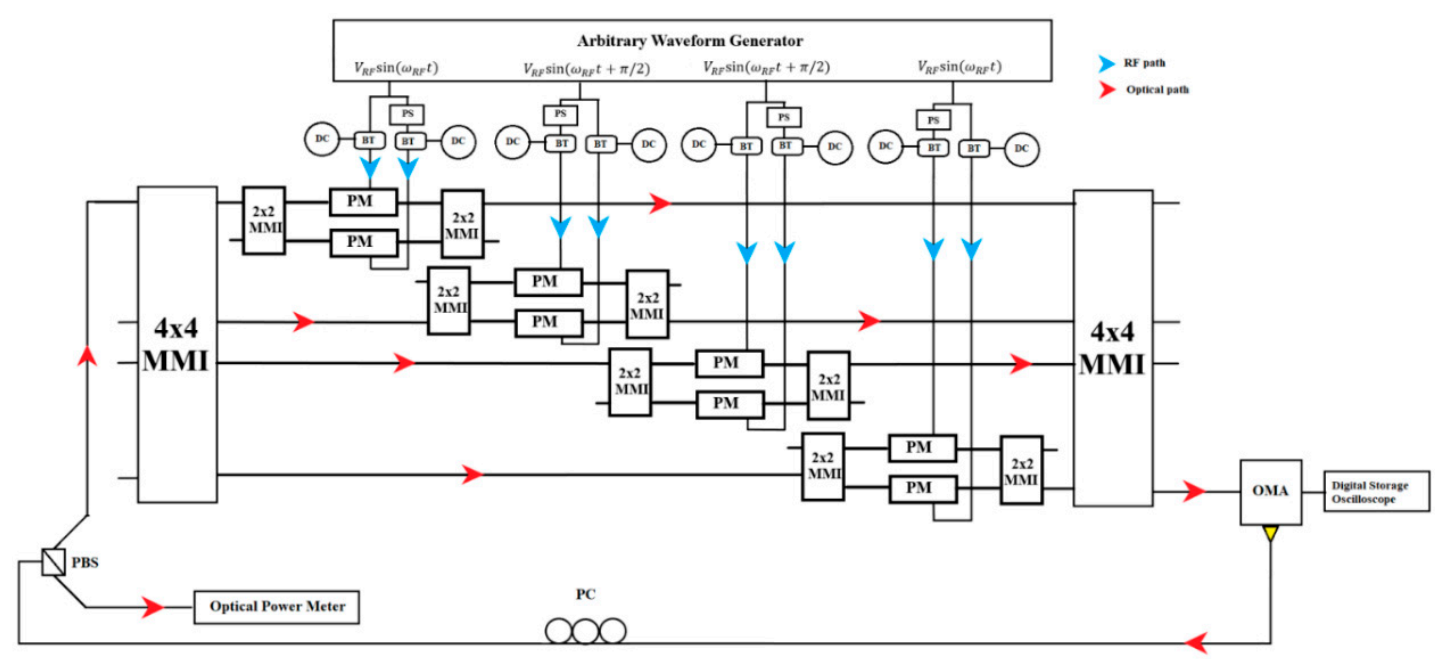

Figure 5. Schematic of the experimental setup. OMA, optical modulation analyzer; PC, polarization controller; PBS, polarization beam splitter; PM, phase modulator; BT, bias tee; PS, phase shifter. The phase modulators are of equal length.

\subsection{Static Assessment}

Due to the absence of test structures on the chip, it is not possible to isolate one MZI and conduct experiments to derive its characteristics. As discussed in Section 2, the circuit provides a degree of freedom where any MZM can be disconnected by applying no or identical modulating signals to its both arms. An experiment is designed based on this feature. A DC voltage is applied to one arm of a single MZI where the other MZIs are biased at null point by design, acting as crossovers. In an ideal situation, light should propagate from input to output through the single MZI which is biased by the DC voltage. Variation of DC voltage should show peaks and notch in its optical transmission spectrum depicting maximum and minimum transmission. Application and variation of the DC voltage to the other arm should result in a contrasting transmission.

Figure 6 shows the variation of optical output power of the circuit due to the variation of DC voltage. For $\mathrm{MZM}_{\mathrm{a}}$, input is applied to the port $\mathrm{I}_{1}$ and output is taken from port $\mathrm{O}_{3}$. For $\mathrm{MZM}_{\mathrm{b}}$, the output port remains the same, but the input port is changed to $\mathrm{I}_{2}$. Both MZMs shows complementary optical transmission characteristics when the DC bias is interchanged between the arms. Significant amount of light at $0 \mathrm{~V}$ indicates that the null point biasing has not been achieved by design only. The off-state, imbalanced MZMs may also contribute which is also reflected in the small extinction. Balanced operation with null point biasing needs precise splitting ratio and phase relationship of MMI couplers and equal path length for the phase modulators. Any minute width deviation in the high contrast SOI platform due to fabrication tolerance or poor design can create huge phase error. So, in practice, for SOI one might as well assume every MZI can be randomly unbalanced. Fine tuning is needed for individual MZM. 
(a)

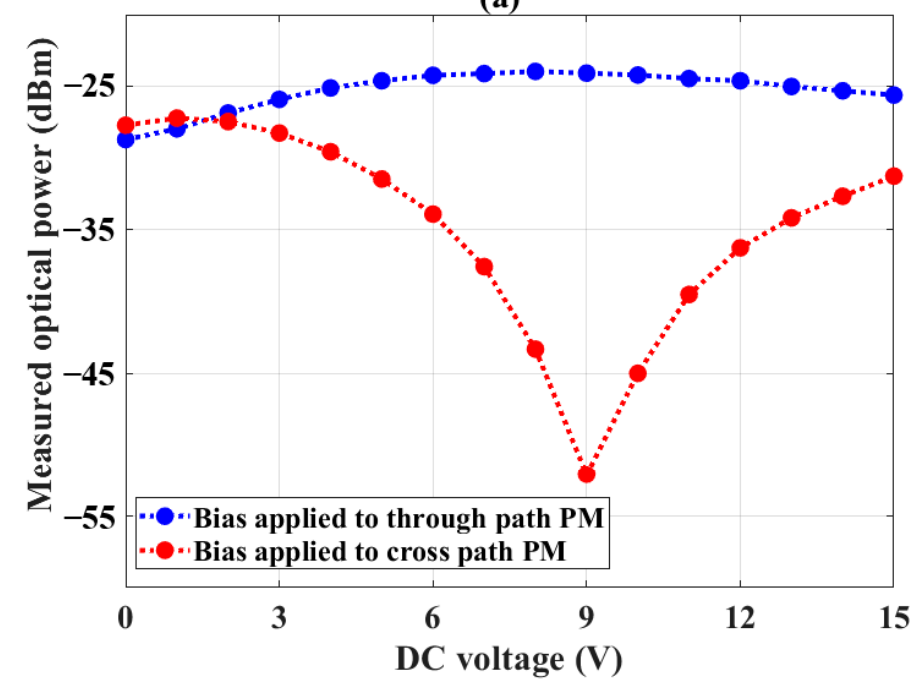

(b)

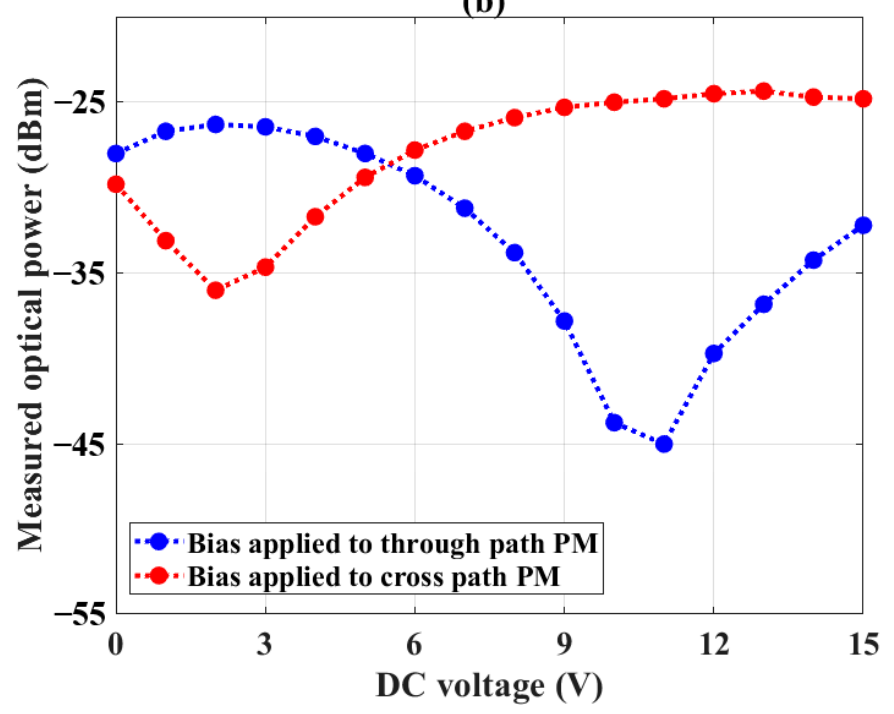

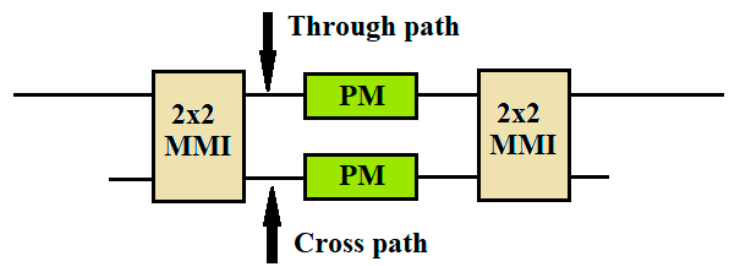

Figure 6. DC characteristics of (a) $\mathrm{MZM}_{\mathrm{a}}$ and (b) $\mathrm{MZM}_{\mathrm{b}}$ in terms of output optical power due to the variation of DC bias voltage. Input port one and two are considered for light input to $\mathrm{MZM}_{\mathrm{a}}$ and $\mathrm{MZM}_{\mathrm{b}}$, respectively. Output is taken from output port three for both cases. Each MZM is asymmetrically driven which means DC voltage is applied to only one arm of the corresponding MZM. PM, phase modulator.

\subsection{Dynamic Assessment}

An ideal differentially driven MZM biased at its null point provides the operation of an intensity modulator. When a pure tone modulating signal is applied to one arm and its $\pi$ phase shifted counterpart to another arm of the MZM, only odd numbered harmonics, spaced by the value of modulating frequency, are retained. Carrier and even order harmonics are completely suppressed without any RF amplitude adjustment. To examine individual MZM of the fabricated circuit, two $10 \mathrm{MHz}$ sinusoidal signals with relative $\pi$ phase difference between them are applied to $\mathrm{MZM}_{\mathrm{b}}$. An arbitrary waveform generator (Fluke 294, Fluke Corporation, Everett, WA, USA) is used as the RF source and the amplitude of the signal has not been adjusted. Figure 7 shows the output optical spectrum. It can be observed that the optical spectrum is corrupted by the presence of carrier and even order harmonics alongside the desired odd order harmonics. Carrier breakthrough reflects the contribution of other off-state MZMs. The imperfect suppression of even order harmonics suggests imbalance in $\mathrm{MZM}_{\mathrm{b}}$. The dual-drive MZM configuration can be utilized to manipulate suppression of unwanted harmonics by individual adjustment of the modulation voltage of each phase shifter. 


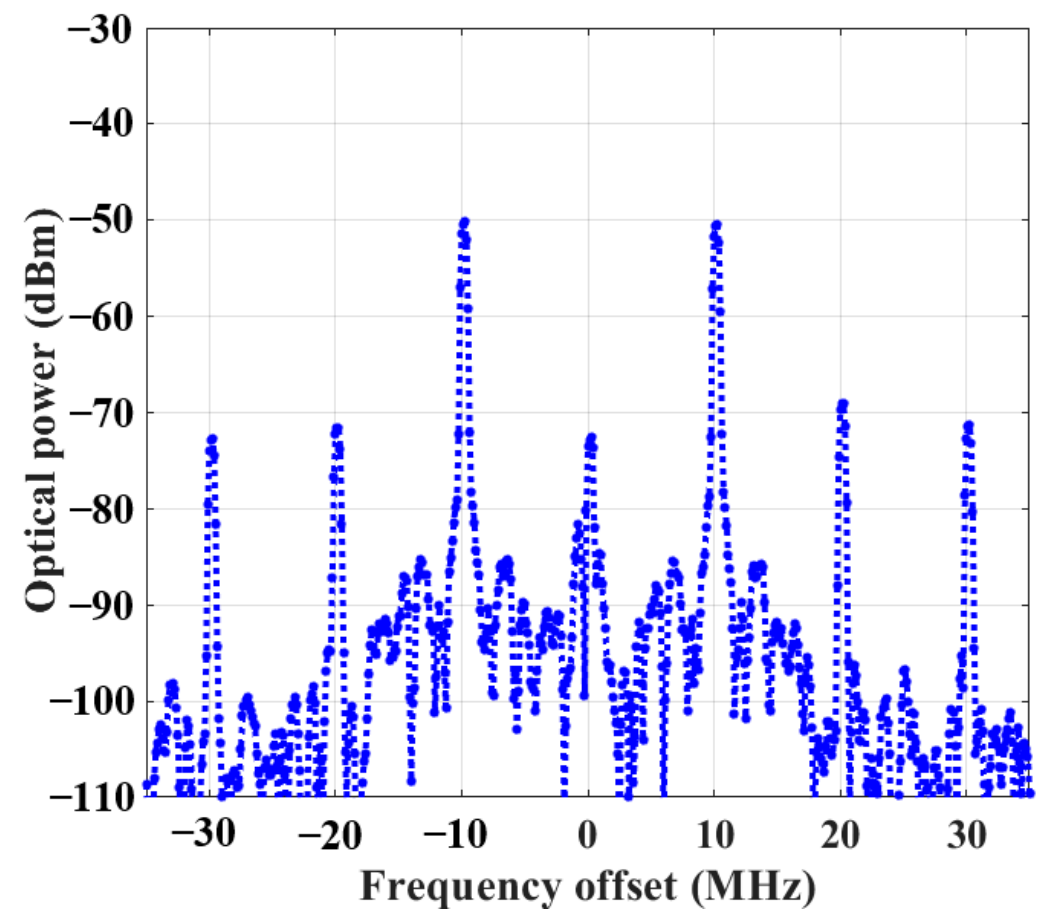

Figure 7. Optical spectrum of the circuit when only $\mathrm{MZM}_{\mathrm{b}}$ is acting as an intensity modulator. The reference frequency is $193.4125 \mathrm{THz}$ and the resolution bandwidth of the OMA is $477.42 \mathrm{kHz}$.

To investigate the frequency shifting capability of the circuit, an experimental setup, as shown in Figure 5, is prepared. RF modulating signals from four channels of the arbitrary waveform generator are applied with the proper relative phase requirement. Although Figure 7 suggests a way to improve the desired harmonics and suppress the spurious harmonics by critically adjusting the RF amplitude and thus the modulation index of each modulator, it needs isolated characterization of individual MZM. Possible phase imbalances in the MMI couplers prohibit this and so, the same RF amplitude is used for all MZMs. Outputs are taken from port one and four while light is launched through input port one. The measured optical spectrums at these output ports are shown in Figure 8. It can be observed that frequency down- and up-conversion is achieved. The carrier breakthrough and appearance of unwanted spurious harmonics limit the circuit's performance predicted theoretically in Equation (6). A carrier suppression of $\sim 20 \mathrm{~dB}$ relative to the desired harmonic with a shifted frequency has been achieved. The most prominent undesired sideband's frequency is also shifted by the same amount as the desired signal, albeit at the opposite direction. A spurious harmonic suppression of $\sim 12 \mathrm{~dB}$ has been measured for both LSB and USB operations.

From the experimental demonstration, it can be conjectured that the deviations from the theoretical prediction can be attributed to imbalanced splitting ratio resulting in finite extinction ratio and phase errors among the ports of the MMI couplers. To circumvent this, tuning mechanisms such as additional phase shifter [27] or integrated variable coupler and variable attenuator [24] can be adopted. A self-adjustment approach described by Miller can also compensate imperfect extinction ratio [34]. Digital signal processing (DSP) enabled solution can also be utilized [29]. Furthermore, broadband sub-wavelength engineered MMI offering a significant reduction in phase error and power imbalance has also been demonstrated $[35,36]$. 
(a)

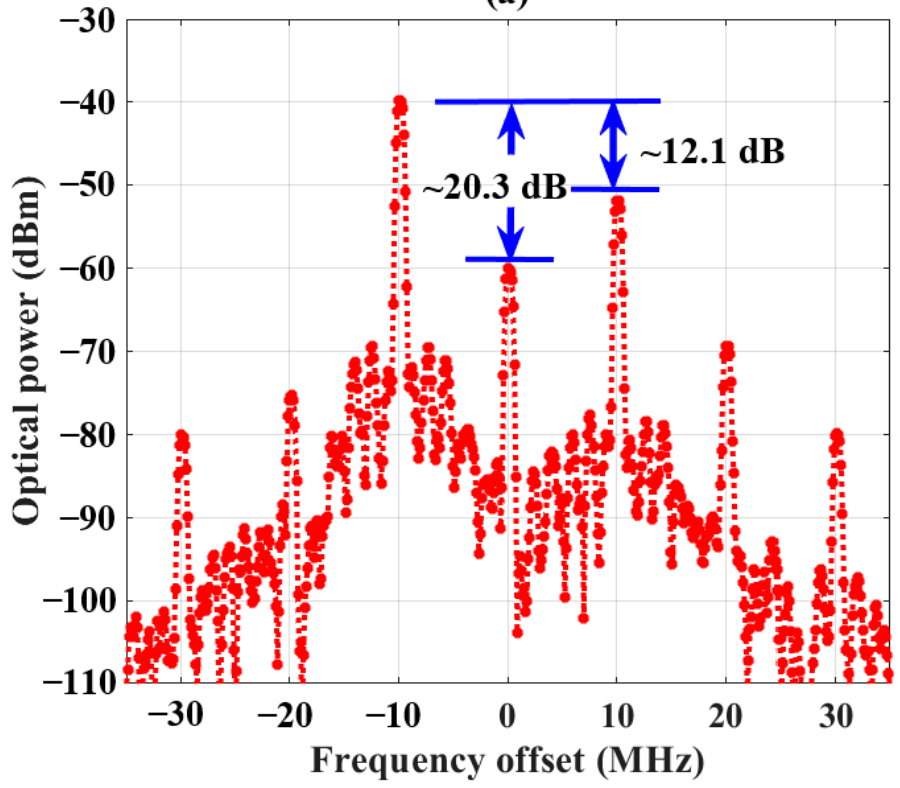

(b)

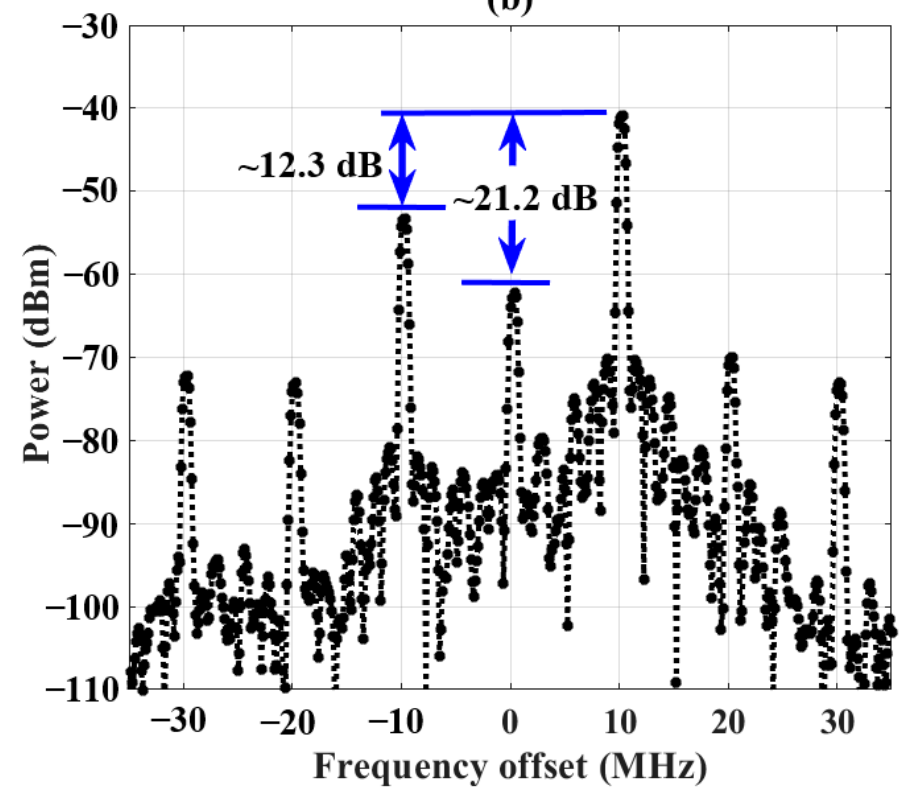

Figure 8. Optical spectra showing (a) frequency down-conversion at output port $\mathrm{O}_{1}$, and (b) frequency up-conversion at output port $\mathrm{O}_{4}$. The reference frequency is $193.4125 \mathrm{THz}$, and the resolution bandwidth of the optical modulation analyzer is $668.388 \mathrm{kHz}$.

High insertion loss $(\sim 20-30 \mathrm{~dB})$ is also limiting the operation. Insertion loss of individual component cannot be measured due to lack of test structure on the fabricated chip. A high index contrast material platform with relatively large sidewall roughness of the narrow ridges can lead to relatively high waveguide propagation loss $(\sim 2-6 \mathrm{~dB})$. A similar loss may be contributed by the doped phase shifters and MMI couplers. The principal contributor to the high insertion loss is the huge coupling loss due to lack of any mechanisms for matching between the access guide mode and the fiber mode ( 12-14 dB). Significant improvement can be attained by adopting a proper coupling mechanism at the access guides $[37,38]$.

\section{Conclusions}

In summary, an optical SSB modulation-based frequency shifter on SOI platform has been demonstrated. Different experiments and observations project imbalanced MMI couplers and high insertion loss to be the limiting factor of the desired performance. A carrier suppression ratio of $\sim 20 \mathrm{~dB}$ and spurious sideband suppression ratio of $\sim 12 \mathrm{~dB}$ has been achieved without any external DC bias for quadrature phase requirement or tuning mechanism for imbalance compensation in MMI couplers. Although the performance has been evaluated by applying $10 \mathrm{MHz}$ RF signal, the low frequency demonstration is imposed by the frequency limit of the available RF generator; the circuit should be capable of operating at the full bandwidth of the modulators $(\sim 10 \mathrm{GHz})$.

The functionality of the fabricated circuit is not limited to optical frequency shifting only. The architecture can also deliver complex modulation, frequency multiplication for millimeter-wave generation, and sub-carrier generation for high data rate transmission using OFDM technology. Any functionality can be implemented by controlling the bias of the MZM and RF phase and thus no modification is needed inside the circuit. These functionalities are being investigated and will be reported in the future. The circuit has been fabricated using 'off-the-shelf' components as a proof of implementation feasibility. To achieve a low loss, low power, spectrally pure, and high bandwidth operation, improvement in component design and fabrication level is necessary. 
resources, G.M.H.; data curation G.M.H. and M.H.; writing-original draft preparation, G.M.H.; writing-review and editing, T.J.H.; visualization, T.J.H.; supervision, T.J.H.; project administration, G.M.H. All authors have read and agreed to the published version of the manuscript.

Funding: The APC was funded by Natural Sciences and Engineering Research Council of Canada (NSERC).

Institutional Review Board Statement: Not applicable.

Informed Consent Statement: Not applicable.

Data Availability Statement: The data that supports the findings of this study are available from the corresponding author upon reasonable request.

Acknowledgments: The fabrication of the circuit was supported by CMC microsystem. The authors would like to show their gratitude to Jessica Zhang, Engineering consultant-photonics at CMC Microsystems for her contribution to mask layout. The authors also thank Ramón Maldonado-Basilio for his help in wire bonding. Mehedi Hasan acknowledges the Natural Sciences and Engineering Research Council of Canada (NSERC) for their support through the Vanier Canada Graduate Scholarship program. Trevor Hall is grateful to the University of Ottawa for their support of his University Research Chair.

Conflicts of Interest: The authors declare no conflict of interest.

\section{References}

1. Hall, T.J.; Maldonado-Basilio, R.; Abdul-Majid, S.; Seregelyi, J.; Li, R.; Antolín-Pérez, I.; Nikkhah, H.; Lucarz, F.; Tocnaye, J.B.; Fracasso, B.; et al. Radio-over-Fibre access for sustainable Digital Cities. Ann. Telecommun. Ann. Télécommun. 2013, 68, 3-21. [CrossRef]

2. Hirooka, T.; Yoshida, M.; Kasai, K.; Nakazawa, M. Optical and wireless-integrated next-generation access network based on coherent technologies. In Proceedings of the Broadband Access Communication Technologies X, San Francisco, CA, USA, 13-18 February 2016; Dingel, B.B., Tsukamoto, K., Eds.; SPIE OPTO: San Francisco, CA, USA, 2016; p. 977203.

3. Kowalski, F.V.; Hale, P.D.; Shattil, S.J. Broadband continuous-wave laser. Opt. Lett. 1988, 13, 622-624. [CrossRef]

4. Cygan, A.; Lisak, D.; Morzyński, P.; Bober, M.; Zawada, M.; Pazderski, E.; Ciuryło, R. Cavity mode-width spectroscopy with widely tunable ultra narrow laser. Opt. Express 2013, 21, 29744-29754. [CrossRef]

5. Li, J.; Li, Z. Frequency-locked multicarrier generator based on a complementary frequency shifter with double recirculating frequency-shifting loops. Opt. Lett. 2013, 38, 359-361. [CrossRef]

6. Li, J.; Zhang, X.; Li, Z.; Zhang, X.; Li, G.; Lu, C. Theoretical studies on the polarization-modulator-based single-side-band modulator used for generation of optical multicarrier. Opt. Express 2014, 22, 14087-14095. [CrossRef] [PubMed]

7. Frankel, M.Y.; Esman, R.D.; Parent, M.G. Array transmitter/receiver controlled by a true time-delay fiber-optic beamformer. IEEE Photon. Technol. Lett. 1995, 7, 1216-1218. [CrossRef]

8. Li, Y.; Meersman, S.; Baets, R. Optical frequency shifter on SOI using thermo-optic serrodyne modulation. In Proceedings of the 7th IEEE International Conference on Group IV Photonics, Beijing, China, 1-3 September 2010; IEEE: Piscataway, NJ, USA, 2010; pp. 75-77.

9. Tanabe, T.; Notomi, M.; Kuramochi, E. Measurement of ultra-high-Q photonic crystal nanocavity using single-sideband frequency modulator. Electron. Lett. 2007, 43, 187-188. [CrossRef]

10. Johnson, L.M.; Cox, C.H. Serrodyne optical frequency translation with high sideband suppression. J. Lightwave Technol. 1988, 6 , 109-112. [CrossRef]

11. Heismann, F.; Ulrich, R. Integrated-optical frequency translator with stripe waveguide. Appl. Phys. Lett. 1984, 45, 490-492. [CrossRef]

12. Shen, Y.; Zhang, X.; Chen, K. Optical single sideband modulation of 11-GHz RoF system using stimulated Brillouin scattering. IEEE Photon. Technol. Lett. 2005, 17, 1277-1279. [CrossRef]

13. Sharma, G.P.; Preußler, S.; Schneider, T. Precise optical frequency shifting using stimulated Brillouin scattering in optical Fibers. IEEE Photon. Technol. Lett. 2017, 29, 1467-1470. [CrossRef]

14. Nosu, K.; Rashleigh, S.C.; Taylor, H.F.; Weller, J.F. Acousto-optic frequency shifter for single-mode fibres. Electron. Lett. 1983, 19, 816-818. [CrossRef]

15. Cummins, H.Z.; Knable, N. Single sideband modulation of coherent light by Bragg reflection from acoustical waves. Proc. IEEE 1963, 51, 1246. [CrossRef]

16. Hui, R.; Zhu, B.; Huang, R.; Allen, C.T.; Demarest, K.R.; Richards, D. Subcarrier multiplexing for high-speed optical transmission. J. Lightwave Technol. 2002, 20, 417.

17. Frankel, M.Y.; Esman, R.D. Optical single-sideband suppressed-carrier modulator for wide-band signal processing. J. Lightwave Technol. 1998, 16, 859. [CrossRef]

18. Xiao, S.; Weiner, A.M. Optical carrier-suppressed single sideband (O-CS-SSB) modulation using a hyperfine blocking filter based on a virtually imaged phased-array (VIPA). IEEE Photon. Technol. Lett. 2005, 17, 1522-1524. [CrossRef] 
19. Zibar, D.; Sambaraju, R.; Jambrina, A.C.; Alemany, R.; Herrera, J.; Monroy, I.T. 16 Gb/s QPSK Wireless-over-Fibre link in 75-110 $\mathrm{GHz}$ band employing optical heterodyne generation and coherent detection. In Proceedings of the 36th European Conference and Exhibition on Optical Communication, Turin, Italy, 19-23 September 2010; IEEE: Piscataway, NJ, USA, 2010 ; pp. 1-3.

20. Izutsu, M.; Shikama, S.; Sueta, T. Integrated optical SSB modulator/frequency shifter. IEEE J. Quantum Electron. 1981, 17, 2225-2227. [CrossRef]

21. Shimotsu, S.; Oikawa, S.; Saitou, T.; Mitsugi, N.; Kubodera, K.; Kawanishi, T.; Izutsu, M. Single side-band modulation performance of a $\mathrm{LiNbO}_{3}$ integrated modulator consisting of four-phase modulator waveguides. IEEE Photon. Technol. Lett. 2001, 13, 364-366. [CrossRef]

22. Yamaguchi, Y.; Kanno, A.; Kawanishi, T.; Izutsu, M.; Nakajima, H. Pure single-sideband modulation using high extinction-ratio parallel Mach-Zehnder modulator with third-order harmonics superposition technique. In Proceedings of the Conference on Lasers and Electro-Optics (CLEO): Science and Innovations 2015, San Jose, CA, USA, 10-15 May 2015; Optical Society of America: Washington, DC, USA, 2015.

23. Yamazaki, H.; Saida, T.; Goh, T.; Mori, A.; Mino, S. Dual-carrier IQ modulator with a complementary frequency shifter. Opt. Express 2011, 19, B69-B74. [CrossRef]

24. Yamazaki, H.; Saida, T.; Goh, T.; Mino, S.; Nagatani, M.; Nosaka, H.; Murata, K. Dual-carrier dual-polarization IQ modulator using a complementary frequency shifter. IEEE J. Sel. Top. Quantum. Electron. 2013, 19, 175-182. [CrossRef]

25. Chow, C.W.; Wang, C.H.; Yeh, C.H.; Chi, S. Analysis of the carrier-suppressed single-sideband modulators used to mitigate Rayleigh backscattering in carrier-distributed PON. Opt. Express 2011, 19, 10973-10978. [CrossRef]

26. Lauermann, M.; Weimann, C.; Knopf, A.; Heni, W.; Palmer, R.; Koeber, S.; Elder, D.L.; Bogaerts, W.; Leuthold, J.; Dalton, L.R.; et al. Integrated optical frequency shifter in silicon-organic hybrid (SOH) technology. Opt. Express 2016, 24, 11694-11707. [CrossRef] [PubMed]

27. Kodigala, A.; Gehl, M.; DeRose, C.T.; Hood, D.; Pomerene, A.T.; Dallo, C.; Trotter, D.; Moore, P.; Starbuck, A.L.; Lee, J.; et al. Silicon photonic single-sideband generation with dual-parallel mach-zehnder modulators. In Proceedings of the Conference on Lasers and Electro-Optics (CLEO): Science and Innovations 2019, San Jose, CA, USA, 5-10 May 2015; Optical Society of America: Washington, DC, USA, 2015.

28. Hasan, M.; Hall, T.J. Photonic circuit for high order USB and LSB separation for remote heterodyning: Analysis and simulation. Opt. Express 2015, 23, 25259-25271. [CrossRef] [PubMed]

29. Mao, M.Z.; Giddings, R.P.; Cao, B.Y.; Xu, Y.T.; Wang, M.; Tang, J.M. DSP-enabled reconfigurable and transparent spectral converters for converging optical and mobile fronthaul/backhaul networks. Opt. Express 2017, 25, 13836-13856. [CrossRef]

30. Li, X.; Zhao, S.; Zhu, Z.; Qu, K.; Lin, T.; Pan, S. An optical frequency shifter based on high-order optical single-sideband modulation and polarization multiplexing. J. Lightwave Technol. 2016, 34, 5094-5100. [CrossRef]

31. Xiao, X.; Li, S.; Xie, Z.; Peng, S.; Wu, D.; Xue, X.; Zheng, X.; Zhou, B. Photonic harmonic up-converter based on a self-oscillating optical frequency comb using a DP-DPMZM. Opt. Commun. 2018, 413, 48-53. [CrossRef]

32. Ma, J.; Wen, A.; Tu, Z. Filter-free photonic microwave upconverter with frequency quadrupling. Appl. Opt. 2019, 58, 7915-7920. [CrossRef]

33. Liow, T.Y.; Ang, K.W.; Fang, Q.; Song, J.F.; Xiong, Y.Z.; Yu, M.B.; Lo, G.Q.; Kwong, D.L. Silicon modulators and germanium photodetectors on SOI: Monolithic integration, compatibility, and performance optimization. IEEE J. Sel. Top. Quantum. Electron. 2009, 16, 307-315. [CrossRef]

34. Miller, D.A. Perfect optics with imperfect components. Optica 2015, 2, 747-750. [CrossRef]

35. Ortega-Moñux, A.; Alonso-Ramos, C.; Maese-Novo, A.; Halir, R.; Zavargo-Peche, L.; Pérez-Galacho, D.; Molina-Fernández, I.; Wangüemert-Pérez, J.G.; Cheben, P.; Schmid, J.H.; et al. An ultra-compact multimode interference coupler with a subwavelength grating slot. Laser Photonics Rev. 2013, 7, L12-L15. [CrossRef]

36. Halir, R.; Bock, P.J.; Cheben, P.; Ortega-Moñux, A.; Alonso-Ramos, C.; Schmid, J.H.; Lapointe, J.; Xu, D.X.; Wangüemert-Pérez, J.G.; Molina-Fernández, Í.; et al. Waveguide sub-wavelength structures: A review of principles and applications. Laser Photonics Rev. 2015, 9, 25-49. [CrossRef]

37. Cheben, P.; Schmid, J.H.; Wang, S.; Xu, D.X.; Vachon, M.; Janz, S.; Lapointe, J.; Painchaud, Y.; Picard, M.J. Broadband polarization independent nanophotonic coupler for silicon waveguides with ultra-high efficiency. Opt. Express 2015, 23, 22553-22563. [CrossRef] [PubMed]

38. Picard, M.J.; Painchaud, Y.; Latrasse, C.; Larouche, C.; Pelletier, F.; Poulin, M. Novel spot-size converter for optical fiber to sub- $\mu$ m silicon waveguide coupling with low loss, low wavelength dependence and high tolerance to alignment. In Proceedings of the 2015 European Conference on Optical Communication (ECOC), Valencia, Spain, 27 September-1 October 2015; IEEE: Piscataway, NJ, USA, 2015; pp. 1-3. 